\title{
El Método LEST, Su Aplicación Y Evaluación En Las Prácticas Ergonómicas
}

\author{
María Guadalupe Obregón Sánchez \\ Profesora investigadora, Instituto Politécnico Nacional-UPIICSA, \\ Avenida Té 950. Colonia Granjas México. México
}

Daniel Islas Reyes

Maestro en Ingeniería Industrial-UPIICSA-IPN

\section{doi: 10.19044/esj.2016.v12n35p34 URL:http://dx.doi.org/10.19044/esj.2016.v12n35p34}

\begin{abstract}
There exist many paths so as to carry out ergonomic modifications. Manuals, models and methodologies can be used in order to appropriately handle loads, the design of job positions, continuous physical movements, service areas; as well as the use of techniques for team work cooperation, among others.

The subject-study company is a producer of rubber-metal parts for the automobile industry. It basically depends on the operational work of its employees. This makes it absolutely important to pay attention to the manner in which they perform their activities.

Many companies pay great importance to the production system without keeping in mind the real needs of workers as they are doing their work. The subject-study company is in this very situation.

This paper shows and approach to ergonomics applied to the Mexican industry. In Mexico, there is little evidence of the use of the LEST method. This is an attempt to take the first steps to carry out ergonomic evaluation with the intention of knowing the conditions under which employees are working within companies and organizations in order to provide them with better working conditions.

The main aim of this research is to now the most incidental variables in the workplace that have an impact on the performance of employees so as to assess the impact of such incidences through the LEST method.

Data was collected from 70 workers taking into consideration their surroundings, workload, mental strains, psycho-social aspects, work shifts, and a new variable that is not considered in the method proposed by the Polytechnic University of Valencia, or by the original method. The workers were asked in a section of the questionnaire: "What would you change about your working environment to improve it?”
\end{abstract}


Through observation, we were able to clearly appreciate the physical movements performed by workers as they went along their physical activities at work while registering each and every one of them. Some of the workers were even filmed to observe the repeated movements they performed during their duties. This allowed us to carry out a more in-depth analysis of such movements.

The Statistical Package for the Social Sciences (SPSS) tool was used so as to tabulate all of the input collected through the questionnaires answered by the employees. By doing so, it was possible to know the frequency at which the abovementioned incidences occurred, and so an interpretation of the data was carried out.

Finally, the results or conclusions obtained through this study revealed that the company has major ergonomic differences between the workload and the work environment, specifically the thermal environment, the acoustic environment and the lighting in the workplace. These areas are closely connected with productivity. In general, the company must modify several aspects in the workplace such as their high staff turnover, as well as the full application of current norms, if the company wishes to grow into a leading company in its field.

Keywords: Ergonomics, LEST method, ergonomics application

\section{Resumen}

Existen varios caminos para llevar a cabo modificaciones ergonómicas. Se pueden utilizar manuales, modelos, metodologías para el manejo apropiado de cargas, diseño de puestos de trabajo, movimientos repetitivos, áreas de servicio y técnicas para la cooperación de grupos de trabajo, entre otros.

La empresa caso de estudio es productora de partes de hule-metal para la industria automotriz; depende principalmente del trabajo de los operarios, por lo que es necesario prestar atención a la manera en que desarrollan sus actividades.

La mayoría de las empresas da importancia al sistema de producción sin pensar en las necesidades reales de los empleados al estar ejecutando sus actividades, y la empresa objeto de estudio se encuentra en esta situación.

El presente trabajo muestra un acercamiento de la ergonomía, aplicándola a la industria mexicana. En México casi no hay evidencia de la aplicación del método LEST y, se trata de dar los primeros pasos para que se practiquen evaluaciones ergonómicas que den como resultado conocer las condiciones en que están laborando los trabajadores en las empresas y organizaciones, para brindarles mejores condiciones laborales. 
El objetivo principal de la investigación es conocer las variables de mayor incidencia en el ambiente de trabajo y que impactan en el desempeño de los trabajadores, para evaluar el impacto de la incidencia mediante el método LEST.

Se aplicó una herramienta de recolección de datos a 70 obreros tomando en consideración el entorno físico, la carga física, la carga mental, los aspectos psicosociales, tiempos de trabajo; y una variable nueva que no contempla el método que propone la Universidad Politécnica de Valencia, ni el método original. Se les preguntó a los trabajadores en un apartado del cuestionario, ¿qué cambiarían de su entorno laboral para estar mejor?

Por medio de la observación se apreciaron claramente los movimientos que hacen los operarios mientras desempeñan su labor, tomando nota de cada uno de sus movimientos; también se recurrió a la filmación de algunos trabajadores para ver de manera repetida los movimientos que llevan a cabo y de esta manera hacer un análisis más profundo.

Se utilizó la herramienta Statistical Package for the Social Sciences, (SPSS), que traduciéndolo al español sería: paquete estadístico para las ciencias sociales. Con este paquete se hizo la tabulación de todos los datos proporcionados en los cuestionarios por parte de los trabajadores, y de esta manera se conoció la frecuencia de las incidencias y se hizo la interpretación. Finalmente los resultados o conclusiones que arroja el presente estudio es que la empresa tiene mayores deficiencias ergonómicas en la carga física y entorno físico, específicamente el ambiente térmico, el ambiente sonoro y el ambiente luminoso. Estas áreas son las que están estrechamente relacionadas a la actividad productiva. Y de manera general, la organización requiere modificar varios aspectos como la alta rotación de empleados, la aplicación correcta de la normatividad vigente, si quiere crecer como una empresa líder en su ramo.

Palabras clave: Ergonomía, método LEST, aplicación ergonómica

\section{Introducción}

El presente trabajo llevó a cabo la aplicación y evaluación ergonómica en una empresa manufacturera, la empresa antes de esta evaluación, no tenía ningún estudio ergonómico dentro de sus áreas de trabajo. Tomando en consideración eso, el objetivo de este estudio fue aplicar el método de evaluación ergonómica a través del método LEST (Laboratoire d'Economie et Sociologie du Travail), utilizando la adaptación que hace la Universidad Politécnica de Valencia, España. Es un método práctico de aplicación y evaluación ergonómica para conocer las anomalías laborales que tienen los trabajadores. 
Se aplicó una herramienta de recolección de datos a 70 obreros tomando en consideración el entorno físico, la carga física, la carga mental, y los aspectos psicosociales, así como tiempos de trabajo. Por medio de la observación se apreciaron claramente los movimientos que hacen mientras desempeñan su labor.

Las respuestas vertidas por los trabajadores se evaluaron mediante la puntuación que establece el método LEST, y se analizaron detenidamente los resultados llegando a concluir que la empresa tiene deficiencias ergonómicas en la carga física y entorno físico, puntualmente en el ambiente térmico, sonoro y luminoso; las áreas están muy ligadas a la actividad productiva de la empresa y de manera general requiere modificar aspectos que están generando malestar a sus trabajadores al desempeñar sus tareas.

Podemos decir que el estudio es novedoso, debido a que no se llevan a cabo comúnmente evaluaciones ergonómicas aplicando el método LEST. La ergonomía en México sigue siendo un campo con pocos seguidores reales, es decir, las empresas todavía no le dan una utilidad real o no la incorporan como un punto primordial para mejorar los intereses de todos, pues aparenta ser más un gasto que una inversión. (El ergonomista, 2004).

Los trabajadores mexicanos se enfrentan a enfermedades que limitan su actividad laboral que tienen su origen en el gran estrés que viven y la mala ergonomía del lugar de trabajo. Las enfermedades que más aquejan a los mexicanos son: dolor de espalda, fatiga visual, agotamiento, cansancio, estrés, obesidad y males gastrointestinales. (CNNExpansión, 2010).

El método LEST se desarrolló en 1978 por F. Guélaud, M.N. Beauchesne, J. Gautrat y G. Roustang, miembros del Laboratoire d'Economie et Sociologie du Travail (L.E.S.T). Provence, Francia. Busca evaluar las condiciones de trabajo de la manera más objetiva y global posible, por medio de un diagnóstico final que indica si las situaciones consideradas en el puesto de trabajo son satisfactorias, molestas o nocivas. El método considera variables que intervienen en el puesto de trabajo de manera general y nos da a conocer en primera instancia si es necesario realizar un análisis más profundo con métodos específicos de cada uno de los puestos de trabajo. El objetivo es evaluar todos los factores relativos a la actividad laboral que pueden tener repercusión en la salud física y emocional de los trabajadores. Antes de la aplicación del método, es imprescindible haber resuelto tópicos referentes a la Seguridad e Higiene en el trabajo dado que no son previstos por el método (Universidad Politécnica de Valencia, 2010).

La información que debe recolectarse para aplicar el método tiene un doble carácter: objetivo y subjetivo. Por un lado se utilizan variables cuantitativas como la temperatura o el nivel sonoro y, por otra, es necesario considerar la opinión del empleado respecto a la tarea que ejecuta en el 
puesto para medir la carga mental o los aspectos psicosociales del mismo. Para que el método sea efectivo, se requiere la participación activa del personal (Universidad Politécnica de valencia, 2010).

A pesar de tratarse de un método general no es posible aplicarlo para la valoración de cualquier tipo de puesto. El método se desarrolló inicialmente para evaluar las condiciones laborales de puestos de trabajo fijos del sector industrial, en los que el grado de cualificación requerido para su desempeño es bajo. Algunos factores del método (ambiente físico, postura, carga física) pueden utilizarse para evaluar puestos con un nivel de cualificación elevado del sector industrial o servicios, mientras el área de trabajo y las condiciones ambientales se mantengan constantes.

Se considera novedosa la aplicación del método LEST, en la presente investigación debido a que se anexó una variable más, que no contempla el método que propone la universidad politécnica de Valencia, ni el método original. La pregunta que se agregó en este estudio fue que los operadores opinaran, qué cambiarían de su entorno laboral para estar mejor.

A continuación se muestran algunas tablas que nos presentan como está conformado el método.

Tabla 1: Dimensiones y variables consideradas en la implementación del método LEST, propuesto por la (Universidad Politécnica de Valencia, 2010).

\begin{tabular}{|c|c|c|c|c|}
\hline $\begin{array}{l}\text { ENTORNO } \\
\text { FÍSICO }\end{array}$ & $\begin{array}{l}\text { CARGA } \\
\text { FÍSICA }\end{array}$ & $\begin{array}{l}\text { CARGA } \\
\text { MENTAL }\end{array}$ & \begin{tabular}{|l} 
ASPECTOS \\
PSICOSOCIALES
\end{tabular} & $\begin{array}{l}\text { TIEMPOS DE } \\
\text { TRABAJO }\end{array}$ \\
\hline Ambiente & Carga estática & Apremio & Iniciativa & Tiempo de trabajo \\
\hline Ruido & Carga dinámica & Complejidad & Estatus social & \\
\hline Iluminación & & Atención & Comunicaciones & \\
\hline \multirow[t]{2}{*}{ Vibraciones } & & Minuciosidad & $\begin{array}{l}\text { Relación con el } \\
\text { mando }\end{array}$ & \\
\hline & & & $\begin{array}{l}\text { Identificación } \\
\text { del producto }\end{array}$ & \\
\hline
\end{tabular}

Para realizar el diagnóstico, el método considera 16 variables agrupadas en 5 dimensiones: entorno físico, carga física, carga mental, aspectos psicosociales y tiempos de trabajo. La evaluación se fundamenta en las calificaciones asignadas a cada una de las 16 variables consideradas.

Tabla 2. Sistema de puntuación del método LEST (Universidad Politécnica de Valencia, 2010).

\section{SISTEMA DE PUNTUACIÓN}

\begin{tabular}{l|l}
\hline $\mathbf{0 , 1 , 2}$ & Situación satisfactoria \\
\hline $\mathbf{3 , 4 , 5}$ & Débiles molestias. Algunas mejoras podrían aportar más comodidad al trabajador \\
\hline $\mathbf{6 , 7}$ & Molestias medias. Existe riesgo de fatiga. \\
\hline $\mathbf{8 , 9}$ & Molestias fuertes. Fatiga \\
\hline $\mathbf{1 0}$ & Nocividad \\
\hline
\end{tabular}


A través de los datos recolectados en la observación del puesto de trabajo y el uso de las tablas de puntuaciones se obtienen las evaluaciones de cada variable y dimensión. La posible puntuación que se puede asignar se encuentra entre 0 y 10; como lo muestra la (Tabla 2).

La aplicación del método inicia con la observación de la actividad desarrollada por el empleado en la que se tomarán los datos necesarios para la evaluación. En general, para la toma de datos objetivos es necesaria la utilización de instrumental adecuado como: un termómetro para la medición de temperatura, un luxómetro para la medición de intensidad luminosa, un sonómetro para la medición de niveles de intensidad sonora, un anemómetro para evaluar la velocidad del aire en el puesto, e instrumentos para medir las distancias y tiempos como cintas métricas y cronómetros.

\section{METODOLOGÍA}

Lo que se refiere a la aplicación del método LEST, se han buscado casos prácticos que muestren la eficacia de éste; y con base en la investigación, se observa que no está muy difundido, debido a que no se encuentran trabajos de esta índole fácilmente. Se han llevado a cabo algunos estudios en España, Francia, México, Chile, y Colombia.

Se inició con la búsqueda de publicaciones ergonómicas que se han realizado y que se encuentran publicados en la base de datos de web of Science, encontrando tres publicaciones de ergonomía en español, y 3457 de ergonomía en inglés “ergonomics”. De las 3 publicaciones en español ninguna es referente a la aplicación del método LEST. Y de las publicaciones en inglés "LEST Method” sólo cinco hacen referencia al método, pero no en aplicación industrial del giro de la empresa en estudio.

En la revisión de base de datos REDALYC, se encontraron 12 publicaciones del Método LEST”, en el cual se hacen evaluaciones pero combinadas con otros métodos.

Podemos decir que la ventaja de la presente investigación es que se aplica el método LEST, sin la combinación de otros métodos, y lo novedoso de este estudio radica en que se adicionó una variable nueva que el método de la Universidad de Valencia y el método original no contempla, y que los investigadores consideraron necesaria.

El método LEST fue aplicado a una muestra de 70 obreros, de los cuales 37 son hombres (entre 19 y 59 años, con media de 36.7) y 28 mujeres (de entre 20 y 55 años con una media 38.1). A los 70 obreros se les aplicó la herramienta de recolección de datos que se diseño; ellos se desenvuelven en puestos y áreas diferentes y éstas son: inyección, vulcanizado, troquelado, tornos, almacén, cementado y preformado.

Durante las visitas de trabajo, cada empleado fue observado desempeñando sus actividades. Se determinó el tiempo requerido para 
realizar un ciclo y se registraron los tiempos que permanecían en cada postura conforme a las indicaciones del método, para poder evaluar la carga física. De acuerdo a las características de la actividad de trabajo fueron contestando las preguntas del cuestionario de evaluación que se aplicó. Para medir las condiciones ambientales se utilizaron los instrumentos de medición como el luxómetro, decibelímetro, termómetros y goniómetros. El tiempo que se empleo en la observación de cada uno de los trabajadores osciló entre los 30 y 45 minutos.

Por otra parte, los trabajadores que son ayudantes generales no tienen un puesto fijo, así que, dependiendo de las necesidades, pueden encontrarse en diferentes áreas. Por esta razón, el análisis de cada persona se efectuó en el área en que se encontraban al momento de llevar a cabo la entrevista y ése es el puesto que se toma como referencia para hacer el análisis de datos.

Inicialmente, se pretendió analizar a los empleados actuales para efectuar la evaluación, pero la rotación de personal es muy alta. Como el periodo de evaluación fue alrededor de 4 meses, algunas personas renunciaron y otras nuevas entraron.

El análisis de la carga de trabajo contempló la carga estática y la carga dinámica. La primera se evaluó en función de las posiciones que se mantienen más comúnmente y la segunda tiene que ver con los esfuerzos que resultan de las labores. Las gráficas siguientes muestran los resultados obtenidos. Se identificó qué porcentaje de personas estaban en qué condición de puesto de trabajo y, además se separaron los porcentajes por departamentos, ya que esto nos da mejor visión de lo que está sucediendo en la empresa.

\section{DIMENSIÓN: CARGA FÍSICA}

Podemos observar que del total de la muestra, más del 50\% se encuentra en una situación nociva para su salud (Gráfica 1). En conjunto, carga estática y dinámica, crean las condiciones para poner en riesgo la salud del trabajador. En general, los departamentos más productivos de la empresa son los que generan mayor carga física, vulcanizado y troquelados. Las posturas son mantenidas por largos periodos y, en general, sin descansos. El turno matutino tiene media hora para comer, mientras que el vespertino tiene que estar trabajando todo el tiempo. Todos pueden hacer cortes para tomar agua o ir al sanitario, pero sólo en esos casos está permitido abandonar sus puestos de trabajo.

La mayoría de las labores se hacen de pie, por lo que esto genera una mayor carga estática. El departamento de vulcanizado mantiene posturas con los brazos estirados y ligeramente encorvados y es ahí donde radican sus problemas. El área de troquelados se mantiene con los brazos estirados y sosteniendo peso para introducir las láminas a las máquinas. En 
lavado y desengrasado la postura dominante es la de estar muy encorvado y, además, levantar grandes cargas en esta posición.

Respecto a la carga dinámica, el principal problema está en el abastecimiento a la máquina de material. Hay algunos puestos en los que, para traer materia prima, deben trasladarse a distancias mayores a 3 metros. Esto provoca que carguen grandes cantidades y el peso que transportan sea bastante. Lo anterior va aunado con las tareas que requieren hacer un mismo movimiento para una gran cantidad de piezas, es decir hacer movimientos repetitivos.

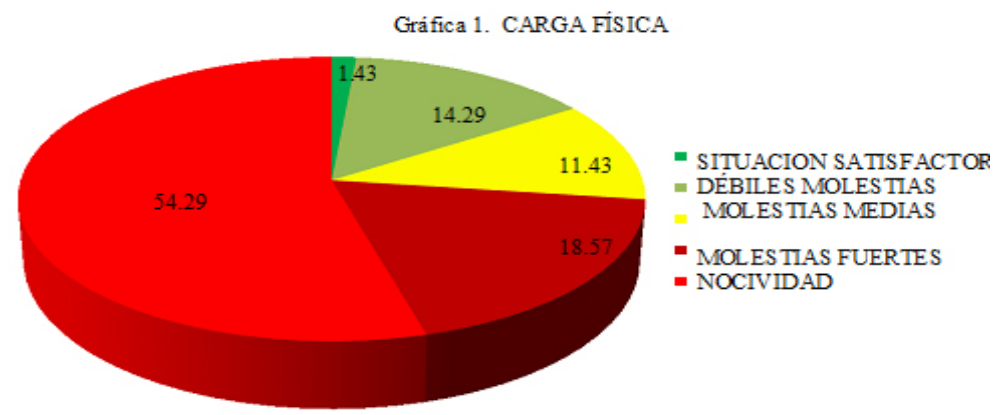

Para estos problemas muchas veces no es posible cambiar las condiciones para que los empleados mantengan posturas más cómodas, ni evitar que se muevan. Lo más viable es proporcionar tiempos de descanso para que el cuerpo se recupere. También es necesario diseñar los espacios de trabajo para que no haya necesidad de trasladarse grandes distancias en busca de material. Junto con esto, es recomendable instruir a los operadores en cómo deben cargar y agacharse y así disminuir el riesgo de lesiones o enfermedades de trabajo a largo plazo.

La carga dinámica requiere el consumo de energía. El turno vespertino no tiene ningún descanso, así que los operadores están sin alimento alrededor de 9 horas de trabajo. El cansancio aparece más rápidamente bajo estas condiciones y el rendimiento de las personas disminuye. Es necesario brindar algún descanso a los trabajadores para que tomen alimento. Se puede inferir que el cambio se dará planeando bien los descansos de los trabajadores.

\section{DIMENSIÓN: ENTORNO FÍSICO}

Respecto al Entorno Físico, los resultados muestran que la mayoría de los empleados están en una situación satisfactoria y con débiles molestias 
(Gráfica 2), lo que es bueno para la planta. Aunque si observamos a detalle, vemos que existen algunos rubros con problemas en algunas áreas, pero que están bien en otras. Esto hace que la situación se equilibre.

En lo que a la temperatura se refiere, los resultados muestran que un $55 \%$ tienen molestias fuertes y con nocividad (Gráfica 3). Estos problemas los encontramos principalmente en el área de vulcanizado. Las máquinas con las que se trabaja, alcanzan temperaturas en los platos de entre 40 y $55^{\circ} \mathrm{C}$. La temperatura que rodea a estos equipos tiene más de $30^{\circ} \mathrm{C}$. Cuando los operadores, están esperando a que vulcanice se encuentran a la última temperatura mencionada. Cuando abren la compuerta y se acercan a los platos para sacar la carga, la temperatura es mayor a los $40^{\circ} \mathrm{C}$. Estos cambios se viven constantemente a lo largo de toda la jornada laboral. Tanto las máquinas de inyección, como de compresión emiten bastante calor. Podemos agregar que los operadores sólo tienen guantes aislantes que, por cierto, no son adecuados y no protegen efectivamente contra quemaduras.

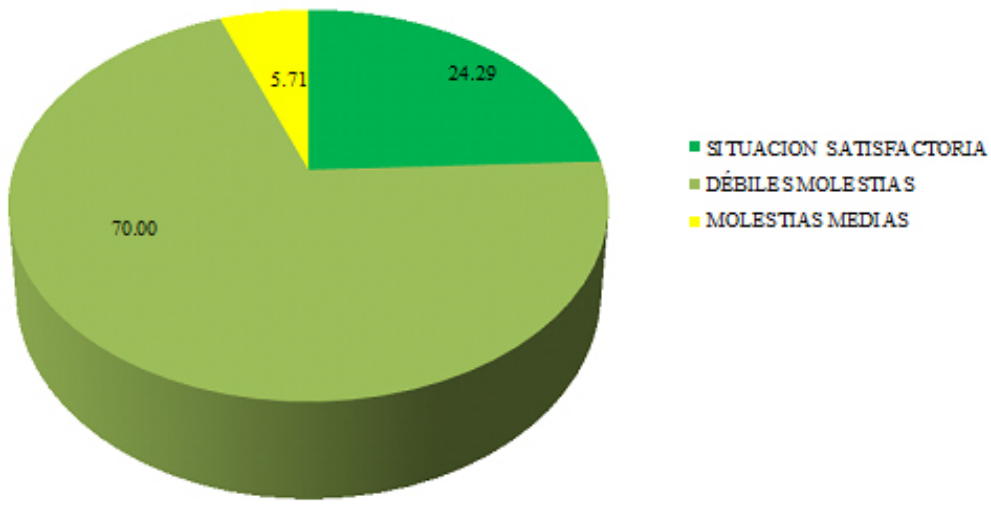

Gráfica 3: AMBIENTE TÉRMICO

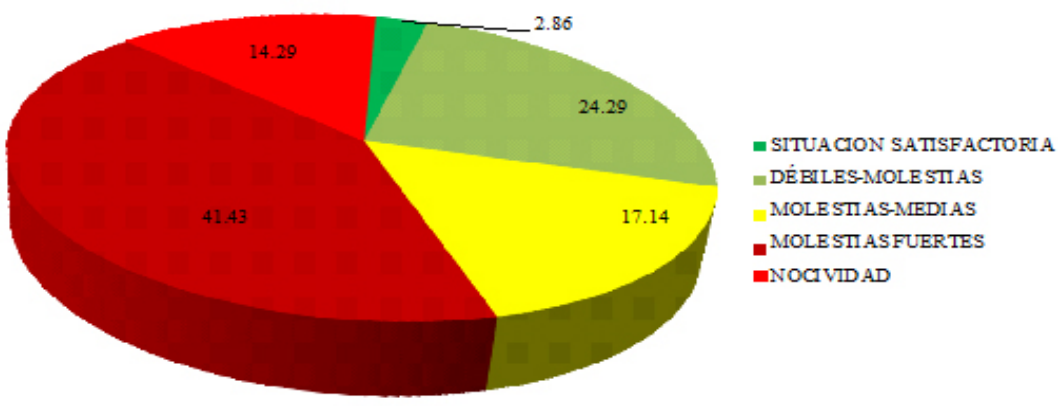


El ambiente sonoro es un aspecto que está dividido, ya que tiene un $45 \%$ de personas que están en situaciones satisfactorias y otro $45 \%$ de personas que están entre molestias fuertes y nocividad (Gráfica 4). Los niveles de ruido son aceptables y constantes (entre 75 y $79 \mathrm{~dB}$ ) en la planta de vulcanizado, mientras que en troquelados son fuertes, variables y se dan bastantes ruidos impulsivos. Otra área que ofrece problemas es el departamento de rebabe, ya que utilizan el esmeril y genera ruido de alrededor de los $86 \mathrm{~dB}$. La NOM-011 permite la generación de ruido en un lugar de trabajo hasta $90 \mathrm{~dB}$ por 8 horas, pero el método LEST, lo califica como muy alto. Es importante mencionar que los operadores utilizan tapones auditivos, lo que les minimiza la cantidad de ruido a que están expuestos.

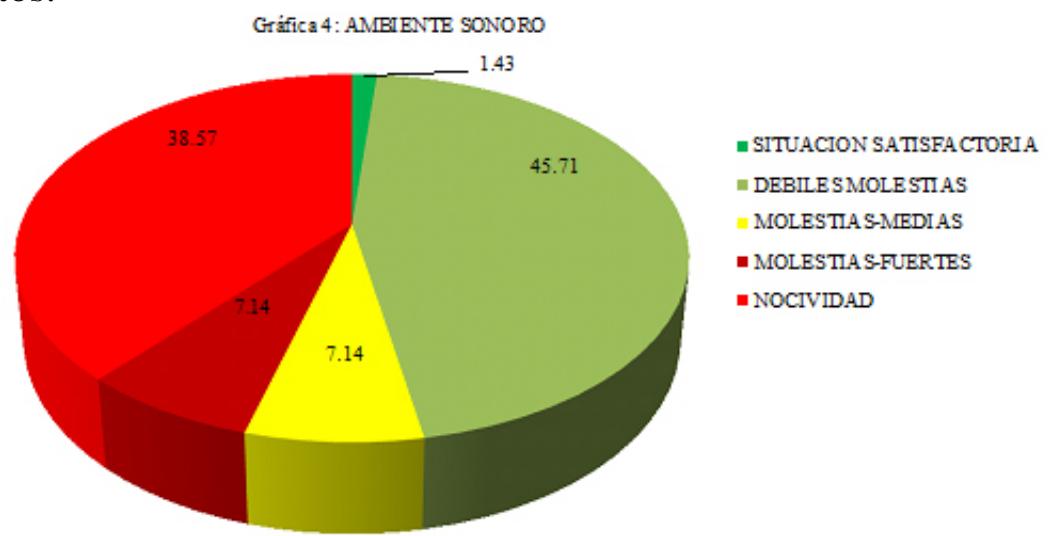

El ambiente luminoso muestra una situación muy favorable para todos los empleados, siempre y cuando las mediciones se hagan cuando hay luz natural. Existen grandes ventanas alrededor de las naves para permitir el paso de luz. En el día existe una media superior a los 500 luxes, la cual es más que suficiente para todas las labores. Casi todas las entrevistas se efectuaron mientras había suficiente luz natural. Las mediciones que se hicieron en las noches mostraron que la luz promedio es inferior a los 100 luxes. A menos que se esté situado exactamente debajo de una lámpara, el valor puede llegar a los 180 luxes. Estos valores no son suficientes para trabajar adecuadamente por la noche. Es necesario agregar lámparas y configurarlas adecuadamente para que haya una correcta iluminación en todos los puntos donde se trabaja.

Respecto a las vibraciones no se encuentran grandes inconvenientes.. La única área que tiene vibraciones es la de tornos y no son molestas. Aunque por el tiempo que se está ahí (todo el turno de trabajo), sí puntúan dentro del método.

Otro aspecto que no viene considerado por este método, pero es importante considerar, es la aspiración de sustancias contaminantes. En este caso, el área de cementado trabaja con solventes como el tolueno y el MIBK, 
éstos son altamente tóxicos y el entorno no tiene la ventilación, ni dispositivos eficientes que se puedan eliminar los vapores.

\section{DIMENSIÓN: CARGA MENTAL}

La Carga Mental se basa en la Presión de Tiempos y en la Atención requerida. En este caso resultó que todos los trabajadores tienes débiles molestias. Los operadores tienen que cubrir cierta cantidad de piezas, así que si se distraen buscan trabajar más rápido. Los supervisores empujan a que cumplan su meta, pero si no la alcanzan no hay mayores repercusiones. Su trabajo no es en cadena, así que ellos van marcando su ritmo.

En cuanto a la atención, los operadores trabajan con piezas medianas y grandes, pueden comunicarse con otros trabajadores $\mathrm{y}$, regularmente sólo operan una máquina. Tienen que mantener su atención por más de 40 minutos y, dependiendo de su actividad, su nivel de concentración va usualmente de débil a medio. Para ayudar a que mejore esta área, se les pueden permitir pausas para que no estén tan atentos por tan largos periodos de tiempo.

\section{DIMENSIÓN: ASPECTOS PSICOSOCIALES}

El $100 \%$ de operadores tuvo un puntaje global en Aspectos Psicosociales de débiles molestias. Esto significa que, en general, es un entorno agradable de trabajo, lo cual coincide con la opinión que tienen los operadores acerca de su ambiente laboral, ya que se les hizo la pregunta directa acerca de este tópico.

En el aspecto de la iniciativa, los trabajadores deciden cómo y a qué tiempo terminan su trabajo, así que esto favorece a que se desenvuelvan como mejor les convenga. Hay tolerancia en cuanto a que algún producto sea irrecuperable, siempre y cuando los operarios no permitan que se siga repitiendo. En su mayoría, importa la habilidad y experiencia del trabajador para que se obtenga el producto terminado, además de que ellos tienen el control de sus máquinas puesto que se les capacita para ello.

Hay gran posibilidad de comunicarse con los demás trabajadores por la corta distancia que hay entre ellos. No se les prohíbe hablar, siempre y cuando estén trabajando. El inconveniente está en que algunas actividades limitan la audición y no es posible hablar unos con otros, así que deben parar la máquina o alejarse de su puesto de trabajo.

Los operadores realizan sus labores tranquilos, ya que en un inicio se les dice qué deben hacer y el supervisor no está vigilándolos constantemente, a menos que tengan pedidos urgentes. Si necesitan apoyo pueden preguntarle a su supervisor, aunque no siempre irá rápido a ayudarles.

El principal problema de esta dimensión está en los periodos de capacitación. Si se les pregunta a diferentes personas en el mismo puesto, la 
gran mayoría difiere del tiempo de capacitación que se les dio cuando ingresaron a la empresa. A su vez, el supervisor menciona tiempo necesarios diferentes. Para esta evaluación, se tomaron los periodos que mencionó dicho supervisor como mínimos necesarios para empezar a trabajar. La mayoría de los operadores normalmente aprende viendo y preguntando a otros, sin que se le brinde una capacitación específica.

\section{DIMENSIÓN: TIEMPOS DE TRABAJO}

En los tiempos de trabajo el $50 \%$ de la población tiene débiles molestias y la otra mitad tiene molestias fuertes. Todos tienen turnos normales y sus horarios son rígidos, así que esto limita un poco a los empleados. La diferencia entre molestias radica en los tiempos de trabajo que laboran. El turno matutino tiene más horas de trabajo, así que su puntuación es más alta. No debía ser así ya que los turnos de trabajo deberían ser proporcionales para evitar sobrecargar y saturar a los operarios de un tuno en particular.

\section{Conclusion}

Existen diversos métodos para realizar evaluaciones ergonómicas pero se estableció que el método LEST era el más adecuado para el tipo de trabajo a estudiar, en función de los parámetros que contempla.

El método LEST, contempla el entorno físico, la carga mental, los aspectos psicosociales y los tiempos de trabajo. Esta metodología muestra una visión más amplia de la situación en la que se encuentra cada empleado, que si se utilizara un método que sólo contempla el esfuerzo por cargas.

El método LEST ha sufrido modificaciones dependiendo de la situación en que ha sido aplicado. La variante que se ocupó en este estudio fue el de la Universidad Politécnica de Valencia. Las posibles respuestas que se tienen para calificar, simplifican mucho el análisis de resultados, en comparación con el método original, ya que los rangos son más amplios.

Como se observa en los resultados, la empresa tiene mayores deficiencias ergonómicas en la carga física y entorno físico en los ambientes térmico, sonoro y luminoso, Estas áreas son las que están más visiblemente ligadas a la actividad productiva. En general, la organización requiere modificar varios aspectos, si quiere crecer como una empresa líder en su ramo.

La iluminación del lugar en el día es buena. De acuerdo a la NOM025-STPS-1999, la cantidad de luz requerida es de 200 y 300 luxes para "requerimiento visual simple: inspección visual, recuento de piezas, trabajo en banco y máquina” y "distinción moderada de detalles: ensamble simple, trabajo medio en banco y máquina, inspección simple, empaque y trabajos de 
oficina”, respectivamente. De acuerdo al método, los niveles óptimos deben estar por encima de estas cantidades, así que es viable aplicar sus parámetros.

Mientras que por el día la iluminación es más que suficiente, por la noche es muy escasa. La norma estipula que iluminación menor a 200 luxes es adecuada sólo para moverse por los pasillos y no para trabajar. La iluminación en las noches oscila entre 150 y 30 luxes, siendo la media de 80. Se recomienda hacer un rediseño del sistema de iluminación, ya que es necesario distribuir adecuadamente las lámparas.

Respecto a los niveles de ruido, encontramos que la NOM-011STPS-2001 marca que el tiempo máximo para una intensidad de $90 \mathrm{~dB}$ es de 8 horas. El método LEST señala que las molestias empiezan a niveles sonoros constantes mayores de $85 \mathrm{~dB}$, por lo que es menos tolerante respecto a la norma. La planta de vulcanizado, en general, no tiene grandes problemas con el ruido, pero la de troquelados tiene ruidos impulsivos repetitivos que sobrepasan los $90 \mathrm{~dB}$. El método establece que ya hay nocividad con estas condiciones.

Las máquinas de trabajo son muy grandes y no existe manera de recubrirlas para disminuir los niveles de ruido, ya que el impacto que se produce en ellas es el generado al cortar la materia prima; esto significa que no es viable hacer una modificación ergonómica.

Los empleados ya cuentan con tapones para los oídos para realizar sus actividades, así que éstos deben ser la medida de prevención que deben continuar usando.

Al referirnos a las temperaturas permisibles, se observa que la planta de vulcanizado es la que tiene mayores problemas. La NOM-015-STPS1993 establece que, por ejemplo, para un trabajo ligero a $32.2^{\circ} \mathrm{C}$ es necesario descansar el 75\% y trabajar el 25\% del tiempo por hora. En la planta de vulcanizado, principalmente, las temperaturas eran similares a este ejemplo pero a condiciones moderadas de trabajo, resultando en condiciones aún más perjudiciales para la salud. Además, el método LEST arrojó resultados esperados de nocividad, ya que la temperatura emitida por las máquinas que se sentía incómoda, coincidió con lo que reportó el método como nocivo.

La carga física tiene puntuaciones altas respecto a la nocividad. De acuerdo a lo observado, la Organización Mundial de la Salud hace algunas recomendaciones, como dar pausas a los operadores, ya que la mayoría está todo de pie durante su turno de trabajo. Se necesita una mejor distribución de las actividades para que estén menos tiempo en la misma posición. Además, se debe capacitar a los empleados para que manipulen las cargas adecuadamente y no vayan a lesionarse y ubicar los materiales siempre cerca de la máquina en la que se trabaja. 
Los aspectos de Carga Mental y Aspectos Psicosociales no reflejan molestias sustantivas para los empleados, de acuerdo a la evaluación con el método, lo que coincide con la actitud que muestran los operadores, ya que abiertamente comentan que el ambiente de trabajo es bueno, que existe apoyo por parte de sus compañeros y las labores no generan gran carga mental.

Existe una alta rotación de operarios en la empresa, así que es difícil rastrearlos. En el periodo que s e llevó a cabo la evaluación, varias personas entraron a trabajar y otras se salieron. Esto puede ser un motivo por el que la empresa no hace caso a las medidas preventivas y de salud, ya que, como la gente renuncia en poco tiempo, no se presenta la situación en que un operador requiera de servicios médicos debidos a malestares ocasionados por la actividad laboral. Aunque se pudiera dar el caso de que alguien se enfermara después de dejar la planta (por causa de las actividades en la planta), sería difícil que lo relacionaran con su verdadera causa.

El método LEST, como otros métodos de este tipo, tiene la desventaja de que algunas variables a evaluar sólo se pueden medir cualitativamente, así que dependerá de quién aplique el cuestionario y la habilidad de éste para obtener la información requerida. Además, las condiciones ambientales que existan en el momento específico en que se hace la entrevista, pueden variar considerablemente en comparación a un mismo puesto de trabajo, por ejemplo: realizar la medición en el día o la noche, en un día nublado o soleado, en primavera o invierno.

Se agregó una pregunta al método original para que los operadores explicaran ¿qué cambiarían de su entorno laboral para estar mejor? Hubo variación de opiniones a esta pregunta, obteniéndose los siguientes resultados.

$\square$ Todo está bien

$30.00 \%$

Mayor mantenimiento a las máquinas

$21.42 \%$

Falta de herramienta y material

$17.14 \%$

Mejor salario

$7.14 \%$

Otros

$24.30 \%$

En función de los resultados, se le debe prestar atención a cómo funciona la maquinaria y si existe material suficiente para que los operadores puedan trabajar mejor.

El método LEST es una buena herramienta para hacer un análisis ergonómico completo. Es recomendable que lo haga una sola persona para que los criterios no cambien. Además, debe llevarse a cabo en un corto periodo de tiempo y dentro de los mismos horarios para que se mantengan más o menos las mismas condiciones del entorno. 
Se llega a la conclusión fundamental, que el método LEST, se puede aplicar a todas las empresas industriales y del giro de la que se evaluó. Sólo cambiarán algunos aspectos tales como la normatividad que rige en cada país para comparar los resultados que arroje el método LEST y la normatividad, para aceptar o rechazar si están dentro de la norma; y llevar a cabo las modificaciones correspondientes para que los trabajadores desempeñen su trabajo en un lugar cómodo y seguro.

\section{References:}

Chandra, A., Chandna, P., Deswal, S. y Kumar, R. (2009). Ergonomics in the Office Environment: A Review. Proceedings of World Academy of Science: Engineering \& Technology. 51, 913-919.

CNNExpansión (2009). Disponible el 8 de noviembre de 2010 en http://www.cnnexpansion.com/mi-carrera/2009/11/11/las-enfermedadeslaborales-mas- comunes.

Cruz, J. A. y Garnica, G.A. (2001). Principios de ergonomía. Colombia: Universidad de Bogotá Jorge Tadeo Lozano.

Domínguez de Hita, R., Hernández Arellano, J. L., García Venegas, E. y García Alcaraz, J. L. (2009). Ergonomic evaluation in driveshaft manufacturing, tool room area. $X V$ Congreso Internacional de Ergonomía SEMAC.

Dzissah, J., Karwowski, W., Rieger, J. y Stewart, D. (2005). Measurement of Ergonomics, 51 (1), 42-48. Cazamian, P. (1986). Tratado de ergonomía. Madrid: Octarés.

Management Efforts with Respect to Integration of Quality, Safety, and ErgonomicsIssues in Manufacturing Industry. Human Factors and Ergonomics in Manufacturing. 15 (2) 213-232.

Ministerio de Trabajo y Asuntos Sociales España, (s.f.). Disponible 11 de mayo de 2011 de http://www.insht.es/InshtWeb/Contenidos/Documentacion/FichasTecnicas/N TP/Ficheros/601a700/ntp_626.pdf.

Modelo, P. R., Gregori, E. y Barrau, P. (2000). Ergonomía 1. Fundamentos. (3ra Ed.). México: Alfaomega

Montmollin, M. (1970). Introducción a la ergonomía. Madrid: Aguilar.

Organización Internacional del Trabajo. (2010). Disponible 1 de noviembre de 2010 deccupati onalsafetyandhealth/lang--es/index.htm

Organización Panamericana de la Salud. (2008). Disponible 1 de noviembre de 2010 de http://www.paho.org/Spanish/DPI/Numero10_articulo1.htm.

Unión Latinoamericana de Ergonomía. (s. f.). Disponible 1 de noviembre de 2010 en http://www.ulaergo.net/quienes_somos.php

Unión Latinoamericana de Ergonomía. (s. f.). Disponible el 1 de noviembre de 2010 en http://www.ulaergo.net/integrantes.php 
Universidad de Buenos Aires. (s. f.) Disponible el 10 de noviembre de 2010 en http://www.fi.uba.ar/archivos/posgrados_apuntes_Metodo_RULA Wisner, A. (1988). Ergonomía y condiciones de trabajo. Buenos Aires: Humanitas. 